\title{
L'agroécosystème et les génies chez les Sèmè du Burkina Faso. Réflexion sur la notion de services écosystémiques culturels
}

\author{
Anne Fournier \\ Institut de Recherche pour le Développement (IRD), UMR PALOC, Patrimoines locaux, environnement et globalisation, Muséum national \\ d'Histoire naturelle, IRD, Sorbonne Université, 43 rue Buffon, Bâtiment 51, 75005 Paris, France
}

\begin{abstract}
Résumé - Cet article montre qu'une lecture des pratiques et des paysages par l'approche des services écosystémiques culturels dans des sociétés traditionnelles se heurte à une incompatibilité des prémisses, celles de l'approche occidentale scientifique étant très différentes de celles des représentations villageoises. La démonstration part d'une pratique de conservation de l'arbuste de jachère Pteleopsis suberosa à des fins culturelles religieuses par les membres de la société sèmè, un groupe de cultivateurs du Burkina Faso. Cette porte d'entrée conduit à mettre au jour différents niveaux de représentations relatifs à la terre, à l'être humain et à l'invisible. Il est montré que si leur attribution à des classes de services écosystémiques est formellement possible, on manque l'essentiel si l'on se contente de cela. L'article discute l'aporie qui consiste à appliquer à une société une construction théorique élaborée selon le mode de pensée d'une autre.
\end{abstract}

Mots clés : services écosystémiques culturels / rituels / représentation de la terre / bosquets sacrés / religion traditionnelle

\begin{abstract}
The agrosystem and the bush spirits among the Sèmè of Burkina Faso. Some thoughts about the notion of cultural ecosystem services. This article shows that reading the practices and landscapes by the means of the cultural ecosystem services approach in traditional societies comes up against an incompatibility of premises, those of the western scientific approach being too different from those of village representations. The demonstration starts from the practice of conserving the fallow treelet Pteleopsis suberosa for religious cultural purposes implemented by the members of the Sèmè society, a group of farmers in Burkina Faso. This point leads to identify different levels of representations relating to the earth, the human being and the invisible. We show that while their allocation to classes of ecosystem services is formally possible, to do only that is to miss the point. The article discusses the aporia of applying to one society a theoretical construction based upon the way of thinking of another society.
\end{abstract}

Keywords: cultural ecosystem services / rituals / representation of the land / sacred groves / traditional religion

\section{Introduction}

Cet article est une réflexion sur la notion de services écosystémiques, il s'appuie sur des recherches relatives aux liens des Sèmè avec la biodiversité végétale et aux représentations qui les sous-tendent. Les dénominations et les divers usages des espèces végétales (Boyd et al., 2014), l'usage religieux de bosquets et de collines (Fournier, 2016), les usages divinatoires des plantes ainsi que les représentations qui y sont associées (Fournier, 2018, 2019) et les pratiques initiatiques ont été documentés. Un essai d'identification d'une conception locale et paysanne des «services écosystémiques » (Bene et Fournier, 2015) a conclu que le principal service

\footnotetext{
*Auteur de correspondance : anne.fournier@ird.fr
}

rendu était d'ordre spirituel : organiser le monde et assurer son harmonie par l'intermédiaire d'êtres invisibles.

Les débats sur les services écosystémiques semblent obscurcis par un quiproquo fondamental. Ce qui entretient l'illusion qu'un tel cadre de lecture est efficient, c'est qu'il permet effectivement de classer certains bénéfices venant de la nature, qui sont connus ou reconnus par les communautés chez qui on l'applique. Ces communautés ayant une connaissance fine de leur environnement, il est en effet possible de renseigner non seulement les catégories d'approvisionnement, mais encore celles de support et de régulation. Une fois cela fait, un reste qui déborde ces catégories subsiste généralement, on le comprend mal et on ne sait trop qu'en faire. Plus le protocole de recueil des données est souple - et mieux il permet donc aux gens de s'exprimer à leur manière -, plus ce reste est copieux. Ce reste est alors généralement affecté à la catégorie 


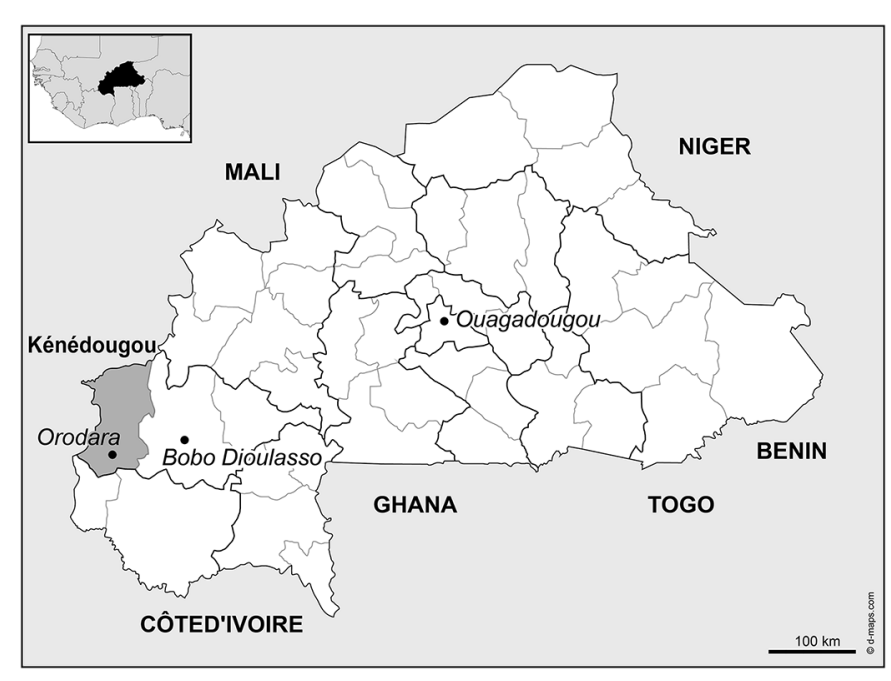

Fig. 1. Localisation des Sèmè au Burkina Faso.

Fig. 1. Location of the Sèmè in Burkina Faso.

des «services écosystémiques culturels», qui ont été définis comme des usages non matériels des écosystèmes, en particulier comme des usages récréatifs, esthétiques, spirituels ou éducatifs (MEA, 2005 ; CAS, 2009). Comme les catégories qui viennent d'être citées se révèlent presque toujours imparfaites ou incomplètes, des ajouts sont faits pour perfectionner la classification, au cas par cas et avec plus ou moins de bonheur. Le «reste» est ainsi réduit autant que faire se peut. La méthode est alors jugée «pas si mauvaise» et l'on estime avoir «respecté » les «communautés locales», parce qu'on les a «fait participer».

Le problème se situe en réalité bien en amont de la question de catégories plus ou moins bien définies ou de celle d'un protocole d'application plus ou moins bien conduit. Le quiproquo fondamental découle de ce que les notions d' " écosystème» et de "nature» sont aussi étrangères à un villageois que celle de «génie de brousse » à un agronome ou à un écologue. Le problème n'est pas seulement de traduction, autrement dit de capacité à identifier dans la langue des gens à qui l'on s'adresse un équivalent suffisamment adéquat des notions d'écosystème ou de nature. Il vient de ce qu'habiter le monde est psychologiquement, sinon philosophiquement, tout autre chose pour les élites instruites - celles qui ont imaginé la notion de services écosystémiques - que pour les communautés rurales - celles à qui l'on applique les questionnaires et qui côtoient quotidiennement des génies. On va tenter de le faire comprendre en s'intéressant au prélèvement d'une espèce végétale de jachère qui intervient dans les pratiques culturelles des Sèmè.

Les Sèmè, population rurale de petit effectif (environ 40000 personnes) et peu connue, vivent au Burkina Faso autour de la ville d'Orodara, dans un environnement naturel de savane (Fig. 1). Sans doute arrivés dans la région au XVII siècle (Fournier, 2016), ils ont longtemps tiré leur subsistance d'une agriculture vivrière de céréales et de tubercules accompagnée d'un peu d'élevage. Depuis une cinquantaine d'années, une arboriculture de rente, qui reste familiale, s'est cependant développée et a fortement modifié paysages et activités (Bene et Fournier 2014, 2015).
Après une courte description des usages mystiques de la plante qui sert à faire les masques portés par les hommes lors de leur initiation, la mise en place d'une protection de cette espèce par les autorités traditionnelles à l'aide de moyens liés à l'invisible va être présentée ainsi que les problèmes auxquels celle-ci se heurte. Les représentations qui motivent les pratiques en vigueur dans la société sèmè seront ensuite expliquées. Enfin, la discussion va se concentrer sur l'aporie que constitue l'emploi d'une notion issue d'un système de valeurs, celle des services écosystémiques, dans un autre système, celui de la pensée des Sèmè.

\section{L'usage matériel religieux d'une plante de jachère}

Les Sèmè restent très attachés à leur religion traditionnelle, le $D$ wo, qu'ils partagent avec d'autres sociétés du Burkina et de Côte d'Ivoire, notamment les Toussian, leurs voisins immédiats, et les Bobo de qui les deux groupes l'auraient reçue. D'après nos entretiens avec les responsables de l'initiation, $50 \%$ des garçons ont subi le rite de passage d'adolescence à Orodara en 2015 et le pourcentage serait encore plus élevé pour les filles. Certains secrets du Dwo sont révélés lors de cette première étape initiatique, mais c'est la cérémonie donoble, organisée tous les 40 ans, qui achève l'initiation masculine, y compris celle des garçons à naître pendant les trois années qui la suivent. Ceux qui n'ont alors pas encore subi le rite de passage parce qu'ils sont trop jeunes ou ne sont pas encore nés le feront à l'adolescence. À Orodara, le dernier donoble a eu lieu en 1984 et le prochain se tiendra en 2024. Chaque village le célèbre à part et leur ordre de passage reflète celui de leur acquisition du Dwo. Après une réclusion initiatique de plusieurs semaines, les nouveaux initiés font une sortie publique couverts de masques représentant des animaux (Petridis, 2008; Bognolo, 2009; Fournier, 2016). Le costume de fibres noires de ces masques (Fig. 2) est fait de l'écorce du petit arbre de brousse à tronc grêle Pteleopsis suberosa (Fig. 3). Chaque cérémonie de donoble nécessite la destruction d'un grand nombre de pieds de cette espèce pour habiller une génération entière.

En termes de services écosystémiques, cette espèce rend donc un service d'approvisionnement à des fins culturelles religieuses. Cet usage peut également être qualifié de «récréatif» car la cérémonie donoble, très appréciée, est également une réjouissance. De plus, le costume des masques a une forte valeur identitaire, car quand d'autres sociétés de la région (comme les Bobo et les Bwaba) habillent aussi certains de leurs masques de robes de fibres, ils emploient une autre plante, Hibiscus cannabinus, qui est cultivée à cet effet.

\section{Mise en protection de la plante, génies et amitié}

Longtemps, l'approvisionnement en Pteleopsis suberosa, espèce extrêmement commune dans la région d'Orodara (Bene, 2011, 2013), n'a posé aucun problème. Ce petit arbre de 7 à $10 \mathrm{~m}$ de haut, qui ne vit qu'une dizaine d'années, affectionne les milieux ouverts sur sols limoneux et graviers fins; il prospère dans les jachères où il devient parfois 


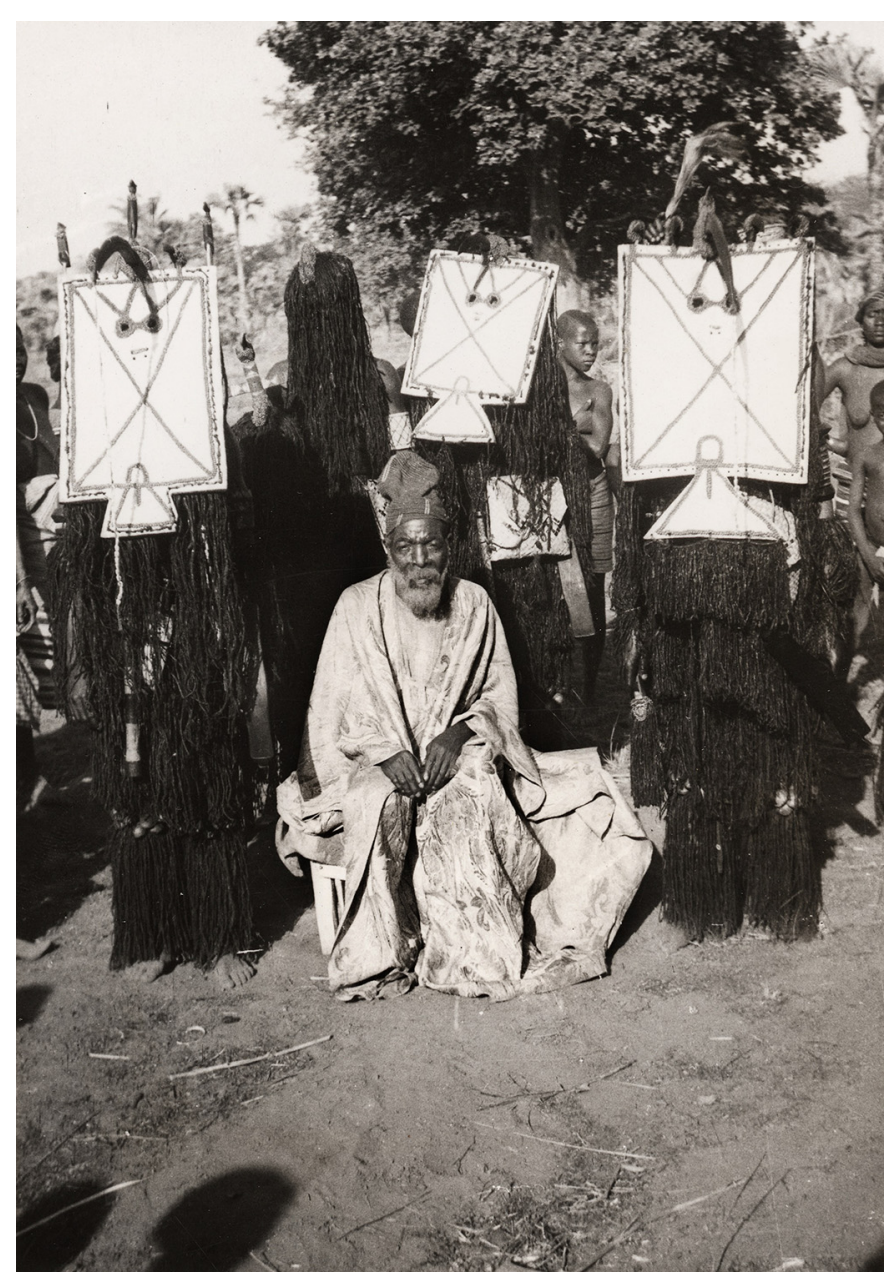

Fig. 2. Masques représentant des calaos avec leurs costumes de fibres de Pteleopsis suberosa lors d'une initiation donoble chez les Toussian (Père Nadal, Toussiamasso, 1950, Archives nationales d'Outre-Mer, droits réservés.).

Fig. 2. Masks representing hornbills with their Pteleopsis suberosa fibre costumes during a Donoble initiation among the Toussian (Father Nadal, Toussiamasso, 1950, Archives nationales d'OutreMer, rights reserved).

envahissant (Aubréville, 1950; Devineau et al., 1997; Arbonnier, 2002). Le mode ancien de mise en valeur tournant des terres, avec essartage, créait autrefois de nombreux espaces de jachère favorables à l'espèce. Avec la croissance démographique et l'introduction, puis l'extension, de l'arboriculture, la superficie des formations naturelles et des jachères a fortement diminué. Ainsi, dans la petite commune de Kotoudéni près d'Orodara, celles-ci sont passées de $76 \%$ du territoire en 1955 à seulement $26 \%$ en 2010 (Bene et Fournier, 2014). Bien avant que la plante ne vienne à manquer, les responsables du culte du Dwo ont décidé de mettre en place des zones où la collecte de l'espèce serait interdite, excepté à l'occasion de donoble. Un marquage par arrachage de l'écorce sur le tronc de certains arbres a été pratiqué sur le pourtour de ces zones. Ce n'est cependant pas par une mise en défens matérielle que les «vieux du Dwo» font respecter l'interdit. L'élément dissuasif est la présence d'un génie sur le site car ce dernier peut causer des problèmes (maladies, malheurs) aux contrevenants ou même les tuer. Lors du choix des sites de défens, les responsables se sont en effet assurés par une divination de la présence d'un génie. Une végétation localement plus dense (Fig. 4) est toujours pour les Sèmè l'indication que le lieu est potentiellement habité par un génie et donc dangereux.

Les espaces de conservation de $P$. suberosa préservent une espèce à usage religieux, mais ils servent de plus de support à un réseau d'échanges sociaux entre villages. Chaque quartier est en effet associé avec un ou plusieurs quartiers d'autres villages; ces derniers lui permettent le prélèvement d'écorces de $P$. suberosa sur leurs terres lors de donoble et y prêtent même la main. Même quand les écorces ne manquent pas, de tels prélèvements croisés sont toujours pratiqués car ces échanges sont prisés pour eux-mêmes, du fait qu'ils entretiennent l'amitié.

Aux services écosystémiques déjà mentionnés, il faut donc en ajouter un autre, celui d'abriter des génies qui font respecter un certain ordre. Doit-on qualifier celui-ci d' «hébergement d'entités invisibles » et de «police forestière religieuse »? Les liens d'amitié entre village autour des mises en défens de l'espèce doivent-ils quant à eux être considérés comme un « service de jumelage entre quartiers »?

\section{Bosquets et autres lieux sacrés chez les Sèmè}

Les bosquets sacrés sont très importants chez les Sèmè et il en existe plusieurs sortes. Le culte du $D$ wo en fait en intervenir deux. Tout quartier possède, au cœur même du village, un bosquet dit «vestibule du Dwo » qui comporte en son centre une maisonnette qui contient des objets sacrés dédiés au $D$ wo (Fig. 5). Les initiés accèdent régulièrement à ce bosquet, notamment pour y pratiquer des sacrifices d'animaux, et chacun est autorisé à le traverser librement sauf pendant les cérémonies. Les hommes s'y présentent obligatoirement plusieurs fois à l'occasion des étapes de leur parcours initiatique. Chaque quartier possède aussi un autre bosquet du Dwo, appelé karmon. Ce lieu «laissé intact» selon la conception des Sèmè est considéré comme un espace de brousse très dangereux et personne ne doit y entrer; seuls le font certains hauts responsables du $D$ wo et seulement à certaines occasions. De très nombreux «bosquets des champs », autre catégorie de bosquets sacrés, parsèment aussi le territoire cultivé. Il s'agit de «bosquets de génies » qui ne relèvent pas directement du culte rendu par les humains au Dwo, bien que les génies soient censés y effectuer leur initiation. Les cultivateurs qui exploitent des champs à proximité de ces bosquets sont tenus d'y effectuer régulièrement des sacrifices propitiatoires. Ainsi, les génies qui y demeurent ne les attaquent pas et n'entravent pas leurs activités agricoles; ils contribuent même au contraire à rendre celles-ci florissantes. Ce sont en effet les génies qui sont les véritables propriétaires du territoire et si les humains peuvent aussi y habiter et y cultiver, c'est parce que les génies leur en accordent le droit.

Dans la communauté des génies, sorte de double invisible de celle des humains, les individus s'initient également au $D$ wo et portent aussi des masques (invisibles); les génies sont donc très concernés par tout ce qui se rapporte à ce culte (Fournier, 2016). 


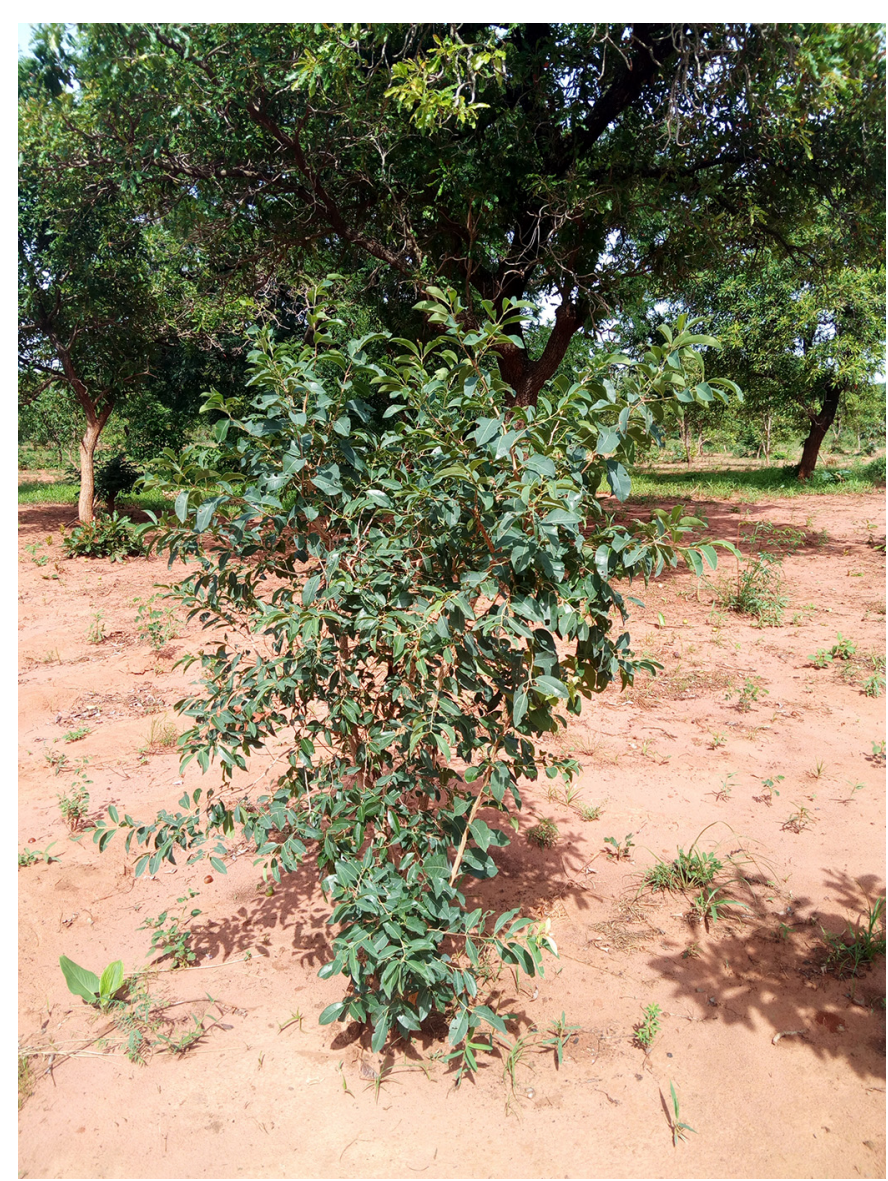

Fig. 3. Jeune individu de Pteleopsis suberosa poussant dans une jachère (Orodara, juillet 2019, (C) Hamadou Coulibaly).

Fig. 3. Young Pteleopsis suberosa tree growing in a fallow land. (Orodara, July 2019, (c) Hamadou Coulibaly).

Les «bosquets de génies » ne sont pas à proprement parler des demeures pour les génies, car ceux-ci vivent dans des collines et des portions de cours d'eau: il s'agit plutôt d'équivalents des «hameaux de culture» des humains, lieux de résidence temporaire où l'on s'installe pendant la période où les travaux des champs battent leur plein.

Ainsi, la brousse peuplée de génies qui offre aux Sèmè un «service agricole» n'est pas un strict équivalent de «la nature » de la pensée occidentale moderne. Comme d'autres sociétés de la région (Bobo, Bwaba), les Sèmè emploient un même terme pour désigner les espaces non ou peu modifiés par les activités humaines et les champs cultivés. Pour eux, la brousse n'est donc pas tant un endroit peu modifié par les activités humaines qu'un espace qui s'oppose à celui du village parce qu'il est placé sous la juridiction des génies et non sous celle des humains, et ceci quand bien même il porte la trace des activités de ces derniers. Cet espace, qui est appelé klon chez les Sèmè, comporte d'autres lieux sacrés que des bosquets. De plus, pour les Sèmè comme pour d'autres sociétés qui vivent en Afrique de l'Ouest et ailleurs, la catégorisation des lieux sacrés ne repose généralement pas sur l'apparence plus ou moins boisée d'un lieu, mais sur la nature de l'entité qu'on y honore (Fournier, 2011). Chez les Sèmè, des collines et des cours d'eau, pourvus ou non de végétation, sont les demeures de génies qui accompagnent la formation des personnes; les premières ont une valence masculine, les secondes une valence féminine. Quand une personne meurt et que son nuon, sorte de principe vital permanent, abandonne son corps, deux génies le guident vers le ventre d'une femme pour qu'il y renaisse. Ce couple de génies provoque la conception, veille sur la grossesse, puis protège le nouvel individu tout au long de sa vie (Fournier, 2016). C'est pourquoi des sacrifices sont régulièrement offerts aux génies des cours d'eau sacrés (inclus dans l'espace villageois) ainsi qu'à ceux des collines sacrées (situées dans la brousse «agricole» ou «sauvage»). Des génies veillent aussi individuellement sur les personnes dans le contexte du Dwo, notamment pendant l'initiation. Ces génies sont associés aux «symboles » animaux du $D w o$, qui à Orodara sont le buffle, la panthère, le chat, la mangouste, le phacochère, le calao et le faucon. Chaque homme initié au Dwo est ainsi rattaché individuellement à un groupe d'appartenance initiatique défini par l'un de ces emblèmes ou «symboles» animaux; il porte le masque correspondant lors de donoble. Ces groupes sont transversaux aux quartiers. Pour « réparer» une «faute de $D w o$ » (toute infraction aux règles du culte), l'initié qui l'a commise doit offrir un sacrifice à une colline où est honoré l'emblème animal correspondant à son « symbole».

Ainsi, les éléments du paysage cultivé ou non offrent aux Sèmè des services non matériels très étendus : réincarnation, conception et naissance, protection individuelle, initiation masculine, ancrage des individus dans divers groupes d'appartenance...

\section{Les limites du dispositif de protection de P. suberosa}

Malgré le dispositif de protection exemplaire instauré par les responsables du Dwo, le gardiennage des réserves de $P$. suberosa par les génies des bosquets n'est pas sans faille. Tant que la pression pour les terres à cultiver n'a pas été trop forte, cette protection a été effective car les bosquets inspiraient une forte crainte. À mesure que cette pression s'est intensifiée, quelques cultivateurs plus audacieux ou plus désespérés que d'autres ont osé, en certains endroits, un grignotage minime de la marge du bosquet. Certains ont été durement frappés: des morts s'en sont ensuivies. Certains autres, encouragés par l'absence de réaction du génie, se sont enhardis et ont poursuivi le défrichement jusqu'à ne laisser qu'une petite bande près de l'autel. L'idée se répand donc que, de nos jours, les génies ne se fâchent vraiment que lorsqu'on ne laisse aucune plante près de l'autel sacrificiel. On chuchote que les vieux responsables, de peur de voir des hécatombes se produire, ont pris les mesures rituelles nécessaires pour que les génies n'attaquent plus les gens qui détruisent les sites de préservation de $P$. suberosa. En plusieurs endroits, surtout dans les quartiers où presque tout le monde est converti à l'islam et au christianisme, toutes les zones de protection de l'espèce ont été "râclées" selon la formule employée localement en français. Sans reconnaître ouvertement une quelconque désactivation des puissances punitives du Dwo par les responsables du Dwo, certains aînés déclarent qu' «on ne peut pas laisser les enfants mourir de faim ». Les vieux du $D$ wo sont en effet très conscients de la dure réalité économique, car s'ils ne cultivent plus eux-mêmes, ils sont les pères et 


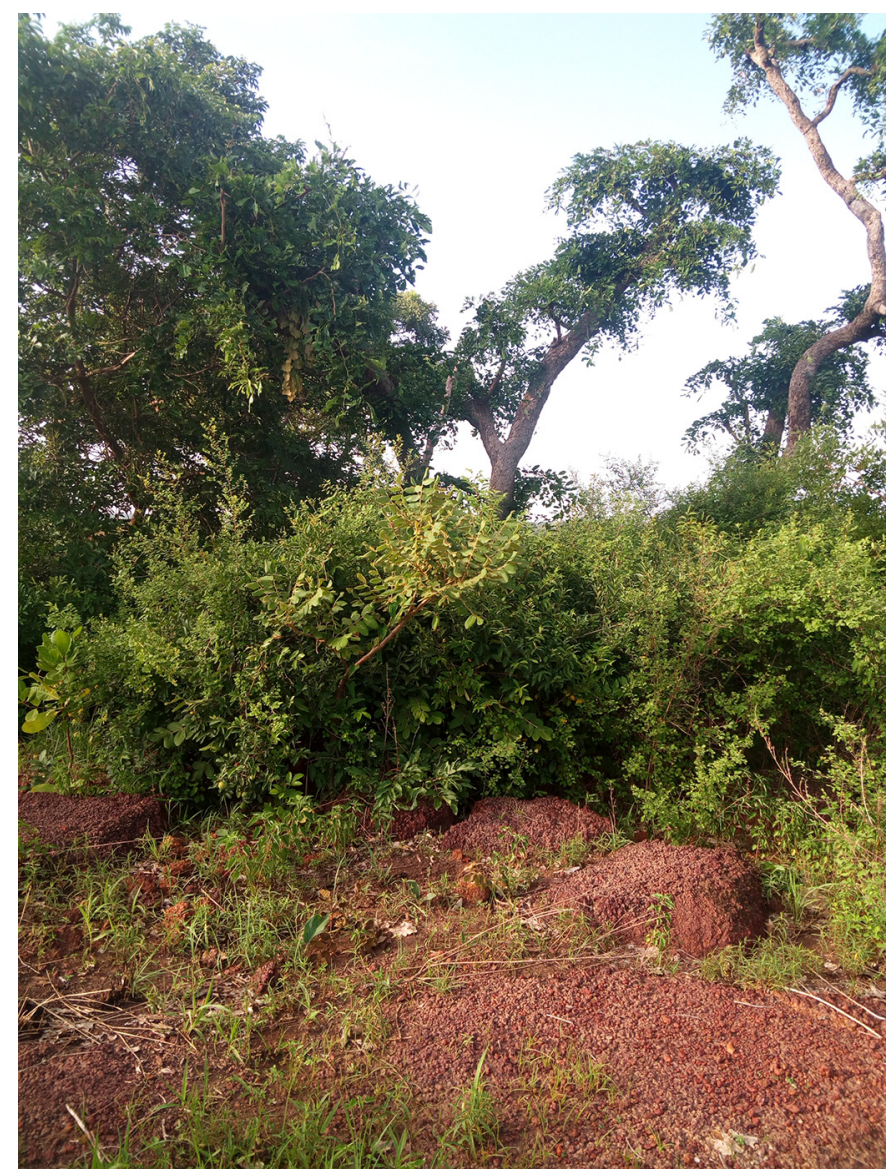

Fig. 4. Site de préservation de Pteleopsis suberosa dans la localité de Diossogo près d'Orodara (juillet 2019, (C) Hamadou Coulibaly).

Fig. 4. Pteleopsis suberosa preservation site in the locality of Diossogo near Orodara (July 2019, (C) Hamadou Coulibaly).

grands-pères des jeunes actifs. Si les convertis sont plus audacieux dans leurs atteintes aux sites de préservation de $P$. suberosa, ce n'est pas qu'ils ne croient plus aux génies, c'est qu'ils pensent que la force de leur nouvelle religion peut les protéger de la vengeance de ceux-ci. Les récits de délivrance d'individus possédés par des génies par des exorcistes chrétiens ou des marabouts musulmans pullulent dans les conversations et dans la presse burkinabé, montrant à quel point les génies restent présents dans les esprits. Une conversion n'implique en effet jamais un effacement simultané complet de toutes les représentations qui constituent les bases d'une culture locale, Langewiesche (2003) l'affirme au sujet du Burkina Faso et M'Biti (1969) pour toute l'Afrique.

\section{Une tradition vivante qui se renouvelle}

L'évolution du monde - « la modernité » comme disent les Sèmè - semble mettre en échec le dispositif de protection de l'espèce $P$. suberosa établi par les responsables du Dwo. La tenue du prochain rituel de donoble est-elle menacée pour autant? Les groupes de responsables du $D$ wo des sept quartiers

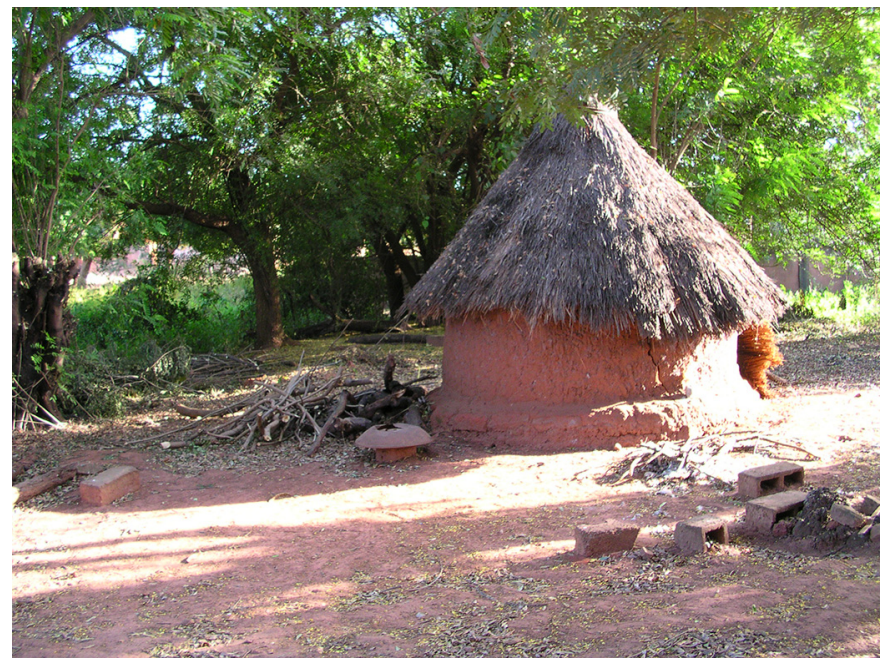

Fig. 5. Dans le bosquet sacré du Dwo, maisonnette contenant les objets sacrés du Dwo dans un quartier d'Orodara (mars 2010, (C) Anne Fournier).

Fig. 5. In the sacred grove of the Dwo, small house containing the sacred objects of the Dwo in a district of Orodara (March 2010, () Anne Fournier).

d'Orodara se réunissent déjà depuis plusieurs années pour la préparer. Ils savent qu'ils procéderont à certains ajustements, car la «tradition» n'est pas une forme immuable qu'on répète aveuglément, mais quelque chose de vivant qui correspond à l'époque où elle se pratique et qui a du sens pour ceux qui la mettent en œuvre. Ils relatent d'ailleurs que des changements ont été introduits entre l'avant-dernier donoble et le dernier. D'après leurs estimations, la quantité de P. suberosa devrait suffire cette fois encore pour «habiller» les prochains initiés. Ils craignent toutefois que cela soit impossible lors du suivant et débattent dès à présent des solutions qui pourront être apportées à ce problème. «Arrêter notre tradition parce qu'une espèce manque ? C'est impensable», déclarent-ils. Il est certes important de reproduire le plus exactement possible ce que faisaient les ancêtres car c'est une forme de respect pour eux, mais quand ce n'est plus faisable, on les consulte par divination sur de nouvelles dispositions et l'on paie le prix rituel qui convient (victimes sacrificielles) pour pouvoir modifier les choses. Peut-être dans l'avenir pourra-t-on utiliser une autre espèce végétale encore disponible pour fabriquer les costumes ? Dans ce cas, elle sera certainement sélectionnée pour ses caractéristiques physiques, mais également en conformité avec la symbolique locale. Peut-être ne fera-t-on plus porter aux masques le vêtement traditionnel de fibres, mais seulement un visage de bois? Ils ne récusent pas ces hypothèses car c'est à l'esprit et non pas à la lettre des rites qu'ils s'attachent. Chez les Bwaba, qui vivent à moins de $200 \mathrm{~km}$ des Sèmè, le problème pourrait être encore plus grave car les masques initiatiques ne comportent pas de visage de bois, mais sont entièrement faits de feuilles fraîches d'arbres d'espèces bien précises. Or, la position des vieux responsables est exactement la même: il faut reproduire exactement ce que faisaient les aïeux tant qu'on le peut, mais si cela devient impossible, on changera les espèces utilisées. 


\section{Discussion et conclusion}

La notion de services écosystémiques relève d'une vision utilitaire (usage de ressources) et rationnelle (phénomènes tangibles), y compris quand il s'agit de services culturels, et les bénéficiaires de ces services sont implicitement considérés comme des consommateurs. Cette vision est antinomique de celle des communautés rurales d'Afrique de l'Ouest, pour qui l'invisible et le sacré se trouvent partout. Dans ses travaux consacrés à la notion de territoire chez les Kasena, autre société de l'aire culturelle voltaïque au Burkina Faso, l'anthropologue Danouta Liberski-Bagnoud (2015, 2019a, b) éclaire la nature du quiproquo évoqué dans l'introduction de cet article. Elle explique que la pensée occidentale envisage la terre comme une chose distincte de ses habitants, postulant que ces habitants «maîtrisent», «gèrent» et se «transmettent» les biens et les ressources qu'elle offre. La notion de services écosystémiques, qui fait l'objet du numéro spécial des Cahiers Agricultures "Agriculture et services écosystémiques dans les pays du Sud» est un excellent exemple de cette position. Toutefois, dans l'aire culturelle voltaïque, notamment chez les Sèmè, le territoire n'est pas pensé comme distinct des gens qui y demeurent, mais comme une sorte de prolongement de leur corps. Ainsi, fonder un village, c'est «instituer un réseau de liens entre des lieux et des lignées, comme entre les lignées elles-mêmes, qui transforme l'espace inhabitable de la brousse en un territoire où les corps humains peuvent se tenir et transmettre la vie propre aux êtres de village» (Liberski-Bagnoud, 2019b). Tant que l'on ne cherche qu'à établir une liste de services, fussent-ils culturels, on reste à la surface des choses puisqu'on manque complètement cette représentation du monde en tant qu'ensemble d'«espacescorps ». Or, en Afrique de l'Ouest, des représentations de ce type sont tout autant présentes dans l'esprit des convertis et des jeunes équipés de smartphones et inscrits à l'université que dans celle des vieux gardiens des traditions. Cette représentation est celle de sociétés parfaitement insérées dans le monde d'aujourd'hui et ce sont des préjugés qui la font considérer comme naïve et déjà presque effacée. Il faut relire les célèbres lignes de Lévi-Strauss (1947) pour se remémorer les raisons qui font qu'une société estime puériles les coutumes d'autrui : «la pensée infantile représente une sorte de commun dénominateur de toutes les pensées de toutes les cultures» écrit-il avant d'expliquer que seuls quelques modèles sont ensuite sélectionnés et maintenus dans la pensée adulte. Ainsi, du fait qu'elle s'exprime dans l'idiome des génies et de l'espace-corps, la pensée religieuse et philosophique des Sèmè par exemple est très difficile à saisir et à prendre en compte pour les membres de sociétés qui sont, au contraire, à l'aise avec la notion de services écosystémiques. Le quiproquo ne peut alors manquer de s'installer.

Tout en décrivant le mode de pensée des Sèmè, nous sommes entrés dans le jeu des services écosystémiques culturels : de nouvelles catégories de services - dont certaines assez plaisantes - ont été proposées. Le lecteur l'aura compris, il s'agit là d'une sorte de démonstration par l'absurde, car toutes les catégories supplémentaires qu'on peut introduire n'empêchent pas la logique des services écosystémiques d'appartenir à une conception du monde qui n'est pas celle des gens chez qui on la met en œuvre. Cette conception est issue d'une culture pour laquelle il est pensable de se limiter à l'aspect économique des choses ou du moins de lui donner la priorité. En toute bonne foi, ceux qui ont recours à la notion de services écosystémiques ne perçoivent pas que cette approche revient à imposer aux gens un mode de pensée venu d'ailleurs. À leur décharge, il faut reconnaître que tant que ce cadre de lecture n'est appliqué qu'aux services non culturels, il semble ne poser que peu de problèmes. Il est cependant patent que tout un pan des réalités locales est alors négligé et c'est pourquoi la notion de services culturels a été ajoutée. Cette solution aggrave sans doute le problème plutôt qu'elle ne le résout car, comme cet article le met en lumière, la démarche revient à occulter, sinon à nier le cadre de lecture du monde de ces gens. On se heurte à une aporie. C'est en effet quand l'approche est « co-construite » avec les membres des sociétés traditionnelles protocole perçu comme le plus vertueux - qu'il est au fond le plus ambigu. Faire participer ces gens à la définition des catégories de services, c'est en réalité les obliger à «se convertir » à un mode de pensée autre que le leur. Le terme provocateur de «se convertir» qui vient d'être employé fera comprendre, nous l'espérons, ce que le procédé peut avoir de violent, voire de pervers si l'on se place du côté de ceux qui y sont soumis. Même si l'on se place sur le plan conceptuel et non sur celui des affects, le problème demeure. Il est en effet très contestable de vouloir appliquer dans un certain système de référence - ici, la pensée des Sèmè- une construction théorique - ici, celle des services écosystémiques - qui a été élaborée au sein d'un tout autre système de référence-ici, la pensée occidentale. Le problème est particulièrement aigu quand les deux systèmes se fondent sur des principes très différents-ici, des conceptions de la terre pratiquement opposées l'une à l'autre. L'efficacité du procédé est même très douteuse. Le cas des services culturels rendus par les jachères chez les Sèmè présenté dans cet article a montré que les catégories des services culturels ne peuvent jamais être définies a priori. Chez les Sèmè, les services culturels qui ont été énumérés n'auraient pas pu être identifiés sans les études ethnographiques menées par ailleurs. Comme le savent les ethnologues de terrain, les Sèmè n'auraient certainement pas évoqué ces éléments dans le cadre d'un questionnement sur les services écosystémiques. Ma propre expérience dans une autre société du Burkina Faso, les Bwaba, peut éclairer ce point. Au cours d'une vingtaine d'années d'études écologiques dans une même localité, donc auprès de gens que je connaissais très bien, je n'avais presque pas entendu parler des génies. Quand j'ai ensuite pratiqué une approche ethnologique, j'ai découvert avec une grande surprise que pour les gens avec qui je travaillais quotidiennement, les génies étaient partout et qu'il s'agissait d'une clé majeure pour comprendre cette société (Fournier, 2011). «Nous n'en avions jamais rien dit parce que nous savions que vous n'y croyez pas et que nous pensions que cela ne vous intéressait pas » m'ont-ils expliqué... Un long cheminement intellectuel m'a ensuite été nécessaire pour saisir un ensemble de notions qui se présentaient d'abord comme fort confuses et fort éloignées de celles que je maîtrisais. La longueur du cheminement s'explique du fait que dans un travail ethnologique, il n'est pas possible de s'en tenir à un domaine et de laisser tout le reste de côté, car tout se tient et un élément éclaire l'autre. Pour expliquer mon ignorance pendant la 
phase écologique de mes travaux, on pourrait, certes, arguer de ma maladresse, de ma fermeture d'esprit, voire de mon mépris pour les gens. Ce type d'imputation alimente une part des éternelles et souvent stériles polémiques entre «naturalistes » et ressortissants des sciences humaines. Il s'agit d'allégations injustes car le décentrage intellectuel indispensable pour accéder au mode de pensée d'une autre culture demande un gros effort et, généralement, beaucoup de temps. Il s'agit d'entreprendre avec persévérance une véritable formation. Pour ceux qui acceptent de jouer les guides, initier un étranger au mode de pensée de sa société représente également un investissement conséquent. Le temps requis pour tout cela est donc incomparablement plus long que celui habituellement dévolu à une étude d'écologie ou d'agronomie. Mon propos n'est donc pas de condamner ceux qui pratiquent l'approche des services écosystémiques et recourent, du mieux qu'ils le peuvent et dans un temps limité, à la catégorie des services culturels. Il ne peut cependant non plus être de livrer des solutions pour améliorer cette approche à laquelle je n'adhère pas. Il s'agit d'amener à mieux identifier la nature du problème que pose l'usage de la notion de services culturels, à percevoir que le «mieux que rien »d'une liste de services culturels établie à l'aide d'une grille de lecture à coloration fondamentalement économique peut donner bonne conscience tout en ayant largement manqué son but.

Remerciements. En la personne de M. Gwene Traore, chef de village d'Orodara, que soient remerciés les nombreux responsables et connaisseurs de « la tradition » qui ont dessillé nos yeux lors de longues conversations. Les travaux ont été financés par le Prix 2018 «Talents de la recherche au Musée de l'Homme » de la Fondation Engie et par les fonds propres de l'unité mixte de recherche IRD/ MNHN PALOC (Patrimoines locaux, environnement et globalisation).

\section{Références}

Arbonnier M. 2002. Arbres, arbustes et lianes des zones sèches d'Afrique de 1'Ouest, $2^{\mathrm{e}}$ éd. Versailles (France): Quae, MNHN, $574 \mathrm{p}$.

Aubréville A. 1950. Flore forestière soudano-guinéenne: AOF, Cameroun, AEF. Paris (France) : Société d'éditions géographiques, $523 \mathrm{p}$.

Bene A. 2011. Évolution de l'occupation des terres et des feux de végétation en pays sèmè. Village de Kotoudéni. Mémoire d'Ingénieur. Bobo-Dioulasso (Burkina Faso): Université de Bobo-Dioulasso, $95 \mathrm{p}$.

Bene A. 2013. Changements globaux et conservation de la biodiversité végétale: valeurs écologique et sociale des espèces et espaces dans le terroir de Kotoudéni (province du Kénédougou, Burkina Faso). Mémoire de DEA. Bobo-Dioulasso (Burkina Faso) : Université de Bobo-Dioulasso, 76 p.

Bene A, Fournier A. 2014. Végétation naturelle et occupation des terres au Burkina Faso (Afrique de l'Ouest): cinq décennies de changement dans un terroir du pays sèmè. In : Fabre G, Fournier A, Sanogo L, dirs. Regards scientifiques croisés sur le changement global et le développement: langue, environnement, culture. Colloque international de Ouagadougou (Burkina Faso), 8-10 mars 2012. Ouagadougou (Burkina Faso): CNRST, pp. 143-164.
Bene A, Fournier A. 2015. Réflexion sur la notion de services écologiques : étude de cas à Kotoudéni (Burkina Faso). VertigO 15 (3). DOI: $10.4000 /$ vertigo.16758.

Bognolo D. 2009. L'animal au cœur de l'identité : rencontre avec la culture toussian, Burkina Faso. Arts and Culture 10: 102-123.

Boyd R, Fournier A, Nignan S. 2014. Une base de données informatisée de la flore: un outil pour la multidisciplinarité. In : Fabre G, Fournier A, Sanogo L, dirs. Regards scientifiques croisés sur le changement global et le développement: langue, environnement, culture. Colloque International de Ouagadougou (Burkina Faso), 8-10 mars 2012. Ouagadougou (Burkina Faso): CNRST, pp. 165-200.

CAS (Centre d'analyse stratégique). 2009. Approche économique de la biodiversité et des services liés aux écosystèmes. Contribution à la décision publique. Paris (France) : La Documentation française, $376 \mathrm{p}$.

Devineau JL, Fournier A, Kaloga B. 1997. Les sols et la végétation de la région de Bondoukui (ouest Burkinabé) : présentation générale et cartographie préliminaire par télédétection satellitaire (SPOT). Paris (France) : Orstom, 177 p.

Fournier A. 2011. Consequences of wooded shrine rituals on vegetation conservation in West Africa: A case study from the Bwaba cultural area (West Burkina Faso). Biodiversity and Conservation 20: 1895-1910. DOI: 10.1007/s10531-011-0065-5.

Fournier A. 2016. Setting up the first components of the person and its anchoring to the territory among the Seme of Burkina Faso: Services rendered by ecosystems? Environmental Skeptics and Critics 5(3): 37-56.

Fournier A. 2018. Divination with plants in West Africa. J Ethnobiology 38(4): 550-567. DOI: 10.2993/0278-0771-38.4.550.

Fournier A. 2019. Marrying the bush spirits-parents. Personal destiny and divination among the Sèmè of Burkina Faso. Anthropos 114(2): 383-398. DOI: 10.5771/0257-9774-2019-2-383.

Fournier A, Douanio M, Bene A. 2014. Pratique et perception des feux de végétation dans un paysage de vergers. Le pays sèmè (Kénédougou, Burkina Faso). In : Fabre G, Fournier A, Sanogo L, eds. Regards scientifiques croisés sur le changement global et le développement-Langue, environnement, culture. Colloque International de Ouagadougou (Burkina Faso), 8-10 mars 2012. Ouagadougou (Burkina Faso): CNRST, pp. 201-229.

Langewiesche K. 2003. Mobilité religieuse : changements religieux au Burkina Faso. Münster (Allemagne): Lit Verlag, Mainzer Beiträge zur Afrika-Forschung.

Lévi-Strauss C. 1947. Les structures élémentaires de la parenté. Paris : Mouton, 591 p.

Liberski-Bagnoud D. 2015. Les gardiens de la terre. La notion de responsabilité dans les systèmes rituels voltaïques. In : Supiot A, Delmas-Marty M, eds. Prendre la responsabilité au sérieux. Paris (France): Presses Universitaires de France, pp. 55-72.

Liberski-Bagnoud D. 2019a. La face inappropriable de la terre. Une autre façon d'instituer le rapport au sol et aux choses (Afrique de l'Ouest). Revue juridique de l'environnement, Lavoisier HS18 (n ${ }^{\circ}$ spécial): 43-54. Disponible sur https://www.cairn.inforevuerevue-juridique-de-lenvironnement-2019-HS18-page-43.htm.

Liberski-Bagnoud D. 2019b. Terre souveraine, terres cadastrées. In : Koch Piettre R, Journet-Diallo O, Liberski-Bagnoud D, eds. Mémoires de la terre. Études anciennes et comparées. Grenoble (France) : Jérôme Million, pp. 371-394.

M'Biti JS. 1969. African religions and philosophy. Londres (Grande Bretagne)/Ibadan (Nigéria)/Nairobi (Kenya): Heinemann, 290 p. 
MEA (Millennium Ecosystem Assessment). 2005. Ecosystems and human well-being: synthesis. Washington DC (USA): Island Press, $155 \mathrm{p}$.
Petridis C. 2008. Buffalo helmets of Tussian and Siemu Peoples of Burkina Faso. African Arts 41(3) 26-43. DOI: 10.1162/ afar.2008.41.3.26.

Citation de l'article : Fournier A. 2020. L'agroécosystème et les génies chez les Sèmè du Burkina Faso. Réflexion sur la notion de services écosystémiques culturels. Cah. Agric. 29: 25. 